\title{
Disseminated Penicillium marneffei Infection with Fungemia and Endobronchial Disease in an AIDS Patient in China
}

\author{
Zong Zhiyong ${ }^{\mathrm{a}}$ Kang Mei $^{\mathrm{b}}$ Liu Yanbin ${ }^{\mathrm{a}}$ \\ ${ }^{a}$ Department of Infectious Diseases and ${ }^{b}$ Laboratory of Clinical Microbiology, West China Hospital, \\ Sichuan University, Chengdu, People's Republic of China
}

\section{Key Words}

Penicilliosis · Penicillium marneffei $\cdot$ Fungemia • Acquired immunodeficiency syndrome

\begin{abstract}
Objective: To present a rare Chinese case of disseminated Penicillium marneffei infection with fungemia and endobronchial disease in an AIDS patient. Clinical Presentation and Intervention: A 26-year-old policeman who had resided in Guangxi Province, China, for 3 years presented to his county hospital with a 2-month history of high fever and cough. A provisional diagnosis of pulmonary tuberculosis (TB) was made. Accordingly, the patient was treated with anti-TB drugs for 1 month but with no clinical improvement; he was then referred to West China Hospital. Blood and bone marrow cultures as well as a bronchoscopic biopsy were positive for $P$. marneffei. A confirmatory serologic test for HIV was positive. A combination therapy with amphotericin $B$ and itraconazole was instituted, and the patient responded well to treatment. Conclusion: This case shows endobronchial involvement caused by an emerging fungal microorganism. HIV-positive patients with a history of residence in or travel to southern China and a clinical presentation suggestive of TB but responding poorly to anti-TB treatment may have $P$. marneffei infection.
\end{abstract}

\section{KARGER}

Fax +41613061234 E-Mail karger@karger.ch www.karger.com

\section{Introduction}

'Penicilliosis marneffei' is a life-threatening invasive fungal infection caused by Penicillium marneffei, which has been reported predominantly from Southeast Asia and Southern China [1, 2]. In Southeast Asia, especially Thailand, this penicilliosis is recognized as an important AIDS-defining illness [1]. However, in Southern China, most cases of the disease have been identified in individuals not infected with HIV [1, 2]. In 1999, the first Chinese AIDS case with penicilliosis was reported from Guangxi Province [3]. Subsequently, several additional cases have been diagnosed in China.

\section{Case Report}

A 26-year-old man who was born and brought up in Sichuan Province, China, was admitted to the local county hospital with a 2 -month history of cough and high fever. For the preceding 3 years he had worked at a garrison in Guangxi Province. Although sputum examination for acid-fast bacilli was negative, a provisional diagnosis of pulmonary tuberculosis (TB) was made. An empiric 4-drug anti-TB therapy regimen including isoniazid, rifampin, ethambutol and pyrazinamide was instituted for over a month. The symptoms of cough and high fever persisted with no clinical improvement, but a weight loss of $6 \mathrm{~kg}$ without accompanying symptoms of diarrhea was noted. The patient was admitted to Sichuan County Hospital. In accordance with the provisional diagnosis of TB, anti-TB treatment was continued with addition of streptomycin. However, 


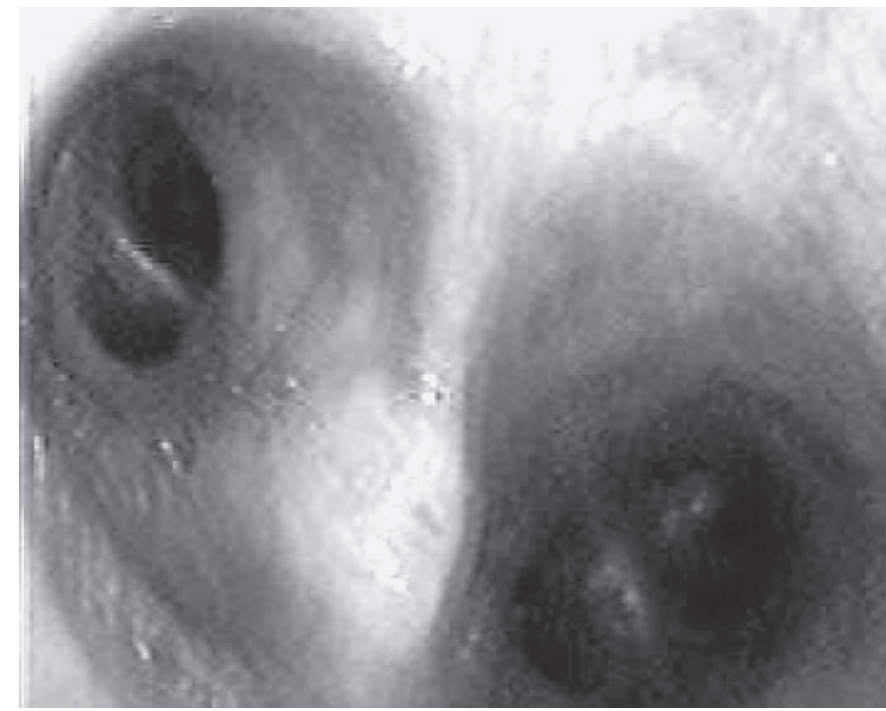

Fig. 1. At bronchoscopy a nodule was found at the wall of the bronchial orifices to the left lower lobe posterior segment.

because of the deteriorating clinical condition, the patient was referred to the West China Hospital, the largest referral hospital in China, for further investigation. The patient was febrile $\left(39.8^{\circ} \mathrm{C}\right)$ with no evidence of skin lesions, lymphadenopathy or hepatosplenomegaly. Findings of respiratory, cardiovascular and gastrointestinal systems were unremarkable. Oxygen saturation on pulse oximetry was $98 \%$ on air and an initial complete blood picture revealed: hemoglobin, $13.4 \mathrm{~g} / \mathrm{dl}$; white cell count, $4 \times 10^{9} / 1$ (neutrophils 3.2 , lymphocytes 0.6 ), and platelets, $62 \times 10^{9} / 1$. Routine serum chemistry was normal and a chest X-ray was reported as normal.

On admission, bone marrow and two blood samples were obtained. After incubation at $37^{\circ} \mathrm{C}$, both blood cultures yielded yeastlike, white and friable colonies with a convoluted surface, but red pigment was not produced. Thus, P. marneffei or Histoplasma capsulatum was suspected. However, following incubation at $25^{\circ} \mathrm{C}$, the colonies developed a downy appearance and there was evidence of soluble red pigment that diffused into the agar, thereby confirming the presence of the microorganisms as $P$. marneffe $i$, and this established the diagnosis of $P$. marneffei fungemia infection.

Fiberoptic bronchoscopy was performed because of persistent cough despite a normal chest X-ray. It revealed endobronchial disease: granulomatous nodules were evident at the bronchial orifices to left lower lobe posterior and basilar segments (fig. 1). Biopsy of these nodules showed a mononuclear cell infiltrate with fungal yeast forms within macrophages, with positive PAS and negative antiacid stains. Thus, a diagnosis of disseminated penicilliosis with endobronchial involvement was established. However, sputum and bronchial lavage fluid were negative on direct microscopic examination for bacteria and fungi, including both acid-fast stained smears and bacterial (including mycobacterial) and fungal cultures. In addition, stained smears and cultures of two bone marrow biopsies were negative for fungal microorganisms.
Only after the patient had been informed of the results of the investigations, he admitted that approximately 1 year earlier, while in Guangxi Province, he had had repeated heterosexual contact with the same female commercial sex worker whose HIV status was unknown. The patient gave informed consent for the HIV screening test. HIV-1/HIV-2 screening tests using two different reagents (Bio Mérieux, France, and Xin Chuang, Xiamen, China) were both positive. The blood sample was referred to the Center for Disease Control and Prevention of Sichuan Province. The HIV-1 confirmatory test using Western blot was also positive. The calculated CD4 count was $8 / \mu 1$ and the CD4/CD8 ratio 0.03 . Thus, a diagnosis of AIDS was established. Additional results of diagnostic tests performed on the patient for CMV, HBV, HCV and syphilis were all negative.

The patient was immediately commenced on combined therapy with intravenous amphotericin B (25 mg once daily) and oral itraconazole (200 mg twice daily) After 3 days, the fever completely resolved. Amphotericin B therapy was stopped after 2 weeks; however, he has been continued on oral itraconazole. To date, the patient has had no recurrence of disease.

\section{Discussion}

We hypothesize that this patient acquired the infection in Guangxi Province located in Southern China prior to his repatriation to Sichuan Province. In China, most penicilliosis cases have been reported from Guangxi Province, which borders on Vietnam. The bamboo rats Rhizomys pruinosus senex and Rhizomys sinensis are the main hosts of $P$. marneffei, and both species are widely distributed in Guangxi Province. In a study in Guangxi Province, P. marneffei was isolated from $96.3 \% R$. pruinosus senex [4]. Although these two species of bamboo rats are also widely distributed in Sichuan Province, environmental studies linking $P$. marneffei and Rhizomys are not reported. Also, there are no reported cases of disseminated penicilliosis acquired locally in Sichuan province.

Penicilliosis may be misdiagnosed as TB [1]. Most cases of penicilliosis have pulmonary symptoms and abnormalities on chest X-ray. As in this case, a misdiagnosis of endobronchial TB may be made, but concern about the validity of the diagnosis should be raised when patients do not respond to medication. Further investigations and a possible diagnosis of penicilliosis may be considered. When TB is suspected, blood culture may not be a priority for some clinicians and their attention is mainly concentrated on the patient's pulmonary illness. Although penicilliosis remains an uncommon diagnosis, under such a clinical presentation, it is important to raise awareness regarding this opportunistic infection. It is obvious from the Chinese medical literature $[2,5]$ that many pen- 
icilliosis cases may initially be misdiagnosed as TB. Thus, physicians and clinical microbiologists should be alerted to the possibility of other diseases including invasive fungal infections among patients with a history of recent travel to Southeast Asia and Southern China who are suspected to have TB but are unresponsive to anti-TB treatment.

The combination of amphotericin B and itraconazole was successful in our case. Although fluconazole controlled some penicilliosis cases [1], amphotericin B alone and/or followed by itraconazole or the combination of amphotericin B and itraconazole is recommended by most studies [1]. The therapeutic success achieved in our case without obvious side effects adds new evidence to this treatment combination as rational therapy for these infected patients.

In the published literature in English and Chinese, endobronchial lesions caused by $P$. marneffei were rarely described. Compared to a previous case with an endobronchial lesion caused by $P$. marneffei which had a positive finding on chest X-ray (a dense opacity in the right upper lobe) [6], the current case was quite different: the lesion sites were at the right upper and left lower lobes for the reported and current cases, respectively. The 2 cases, however, shared some similarities: for instance, both were young Chinese males, stayed in Southern China for a period, had very low CD4 counts, presented with cough and fever, and had a good response to antifungal therapy within 1 week. The patient in the present study did not undergo bronchoscopy on his first hospital admission and was misdiagnosed as having endobronchial TB, but no acid-fast bacilli were visualized on direct microscopic examination. We therefore advocate that endobronchial involvement caused by pathogens other than Mycobacterium tuberculosis ought to be considered, especially when the patient has negative chest radiographic findings and is febrile with a high temperature.

\section{Conclusion}

Generally, penicilliosis marneffei is an emerging opportunistic fungal infection associated with AIDS. The disease is usually associated with a history of residence in or travel to Southeast Asia and Southern China. P. marneffei may cause endobronchial involvement. Bronchoscopy and bronchial biopsies are essential for confirming the diagnosis in patients with this infection. Amphotericin B combined with itraconazole is considered the therapy of choice for the infection.

\section{Acknowledgement}

We thank Dr. Michael M. McNeil, Epidemiology and Surveillance Division, National Immunization Program, CDC, USA, for his urgent review and editing this article.

\section{References}

1 Duong TA: Infection due to Penicillium marneffei, an emerging pathogen: review of 155 reported cases. Clin Infect Dis 1996;23: $125-130$.

$>2$ Deng ZL, Ribas JL, Gibson DW, Conor DH: Infections caused by Penicillium marneffei in China and Southeast Asia: review of eighteen published cases and report of four more Chinese cases. Rev Infect Dis 1988;10:640-652.

3 Deng ZL, Liu XM: Disseminated penicilliosis marneffei in a patient with acquired immunodeficiency syndrome: a first case report from China. Chin Med J (Engl) 2000;113:10491050.
4 Li S, Deng ZL, Ma Y: The study on the carriage of Penicillium marneffei in Guangxi's rats. Guangxi Med 1995;17:1-2.

5 Pan J, Li JS: Analysis of 12 cases of penicilliosis marneffei. Chin J Dermatol 1999;32:353.

6 McShane H, Tang CM, Conlon CP: Disseminated Penicillium marneffei infection presenting as a right upper lobe mass in an HIV-positive patient. Thorax 1998;53:905-906. 